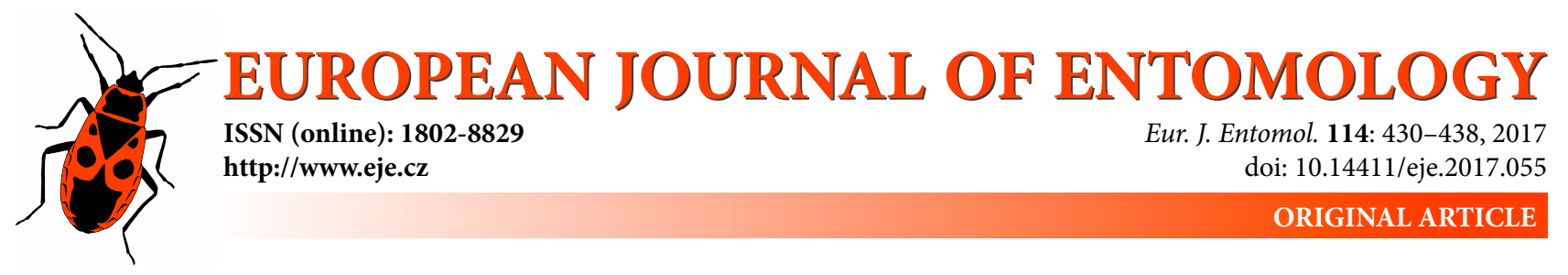

\title{
Characterization and functional analysis of the serpin-10 gene from oak silkworm, Antheraea pernyi (Lepidoptera: Saturniidae)
}

\author{
Saima KaUSAR*, Cen QIAN*, Muhammad Nadeem AbBaS*, Bao-Jian ZHU, Ya LiU, Lei Wang, Guo-Qing Wei, Yu SUN \\ and ChAO-LIANG LIU**
}

College of Life Sciences, Anhui Agricultural University, 130 Changjiang West Road, Hefei, 230036, China; e-mails: drkausarsn@hotmail.com, qiancenqiancen@163.com, abbasmndr@gmail.com, zhubaojian@ahau.edu.cn, 243685181@qq.com, wanglei20041225@163.com, weiguoqing@tom.com, nagato0621@163.com, anhuicansang@163.com

Key words. Lepidoptera, Saturniidae, Antheraea pernyi, serpin, innate immunity, haemolymph protein

\begin{abstract}
Serpin is a broadly distributed superfamily of proteins that have a crucial role in regulating various immune reactions. Herein we identified a serpin-10 gene from Antheraea pernyi that encodes a 1557 amino acid residue protein with a predicted molecular weight of $58.76 \mathrm{kDa}$. Recombinant Apserpin-10 protein was expressed in a prokaryotic expression system (Escherichia coli) and the purified protein was used to prepare rabbit anti-Apserpin-10 polyclonal antibodies. Quantitative real-time polymerase chain reaction and western blot analysis indicate that Apserpin-10 was transcribed in all the tissues examined, including haemolymph, malpighian tubules, fat body, silk gland, integument and mid gut; the greatest expression level of Apserpin-10 was recorded in the fat body and haemocytes. The comparison of different developmental stages showed that Apserpin-10 transcript level was highest in 5th instar larvae, while the lowest expression was recorded at the egg stage. We also investigated the expression patterns of Apserpin-10 in fat body and haemocyte samples, following administration of heat-inactivated gram-positive bacteria (Micrococcus luteus), gram negative bacteria (Escherichia coli), a fungus (Beauveria bassiana) and virus (nuclear polyhedrosis virus, NPV). A substantial up-regulation of Apserpin-10 expression was recorded following pathogen challenge in both the tissues tested. Further the knock down of Apserpin-10 led to down regulation of antimicrobial peptide genes. Altogether, our results indicate that Apserpin-10 is involved in the innate immunity of $A$. pernyi.
\end{abstract}

\section{INTRODUCTION}

Serpin is a widely distributed superfamily of proteins. A variety of serpin and serpin-like genes are documented in both prokaryotes and eukaryotes, and according to estimates this family contains more than 1,500 members (Irving et al., 2000; Rawling et al., 2004). They mainly regulate serine and cysteine proteases in cells. They constitute a single chain, which forms a specific structure with a reactive center loop (RCL) close to the C-terminus, which provides a surface to bind with a target protease (Rawlings et al., 2004). The proteolytic inhibition starts by the formation of a complex between serpin and its target protein (Potempa et al., 1994). In mammals, serpins regulate several physiological processes, by activating proteolytic cascades, such as inflammatory as well as acute phase immune responses (Gooptu \& Lomas, 2009). In humans, protease inhibitor 10 plays a key role in the regulation of protease activity during haematopoiesis and apoptosis induced by TNF (Schleef \& Chuang, 2000).
Many serpins are also described in insects (Reichhart, 2005; Suwanchaichinda \& Kanost, 2009; Zou et al., 2009) and biological studies indicate, as in mammals, that insect serpins also have a wide range of physiological functions and non-inhibitory serpins also have a crucial role in the biology of insects (Gubb et al., 2007). Although, serpins have been identified in the immune system of a few insects, for most insects their role is not fully understood. Currently, serpins are well studied mainly in moths, flies and beetles and most of them regulate several immune reactions (De Gregorio et al., 2002).

Invasion of microbes into the haemocoel affects serpin production (Bulet et al., 1999; Abraham et al., 2005) e.g., the levels of serpin-1 transcripts in the integument of $O s$ trinia furnacalis is inhibited following challenges with Staphylococcus aureus and Escherichia coli (Zhang et al., 2016). In Bombyx mori serpin 15 transcript is recorded in the fat body following a microbial challenge (Liu et al., 2015). In Manduca sexta, B. mori and Drosophila mela-

\footnotetext{
* These authors contributed to this work equally.

** Corresponding author; e-mail: anhuicansang@163.com
} 
nogaster serpins inhibit haemolymph proteases (HPs), which following tissue damage and infection with pathogens, have a role in defence (Kanost et al., 1999). The above results demonstrate that serpins have an important role in the innate immunity of insects.

Antheraea pernyi (Lepidoptera: Saturniidae) is one of the most important silkworm species and of great economic importance in China, India and Korea. Its larvae, pupae and adults contain high quality protein, which is made up of all the amino acids required by the human body (Yang et al., 2002; Zhou \& Han, 2006). It spends its whole life cycle in the wild, so it is more likely to encounter a variety of harmful microbes. To survive in these conditions they have over time evolved an efficient immune system. As this species is of economic importance researchers have studied the immune mechanisms and factors involved in its immune system (Li et al., 2005; Wang et al., 2013; Youlei et al., 2013; Lu et al., 2015). So far, our knowledge of insect serpins and those of $A$. pernyi in particular is limited, with only three serpins studied: serpin 3 (Wang et al., 2017), serpin 14 (Kausar et al., 2017) and serpin 1 (Yu et al., 2017). The aim of this study is to characterize, clone and explore the immune functions of Serpin-10 from A. pernyi. This basic knowledge might help in the further exploration of the immune functions of the Apserpin-10 gene.

\section{MATERIAL AND METHODS}

\section{Rearing of $A$. pernyi}

Sericulture Research Institute at Liaoning, China provided pupae of $A$. pernyi. The pupae were then used to rear adults. Eggs were collected and incubated, and the larvae were fed on fresh oak leaves and reared at room temperature $\left(21-25^{\circ} \mathrm{C}\right)$, under a $10 \mathrm{~L}: 14 \mathrm{D}$ photoperiod, and $70 \%$ relative humidity until they transformed into pupae. In our laboratory, larvae were reared in hundreds (Approximately more than 500) and were randomly selected for the experiments

\section{RNA isolation and cloning of Apserpin-10}

The fat body of larvae of $A$. pernyi ( $5^{\text {th }}$ instar $3^{\text {rd }}$ day) was ground up and the Trizol Reagent (Takara, Dalian, China) used to extract total RNA, which was used to synthesize first-strand cDNA by TransScript Synthesis SuperMix (TransGen, Beijing, China). To amplify Apserpin-10 ORF, gene specific primers (Apserpin10-F and Apserpin10-R) were designed (Table 1). Polymerase chain reaction (PCR) using the first strand cDNA was performed to amplify the desired sequence. PCR conditions were as follows: $4 \mathrm{~min}$ at $94^{\circ} \mathrm{C}$; followed by 35 cycles of $94^{\circ} \mathrm{C}$ for 30 $\mathrm{s}, 51^{\circ} \mathrm{C}$ for $30 \mathrm{~s}, 72^{\circ} \mathrm{C}$ for $1.5 \mathrm{~min}$ and a final elongation step of $72^{\circ} \mathrm{C}$ for $10 \mathrm{~min}$. The PCR products were resolved on $1 \%$ agarose gel, purified using DNA Gel Extraction Kit (Axygen, Hangzhou, China) and sequenced by Invitrogen.

\section{Sequence analysis}

Conserved SERPIN domains in Apserpin-10 were analyzed using NCBI blast tools (http://blast.ncbi.nlm.nih.gov/Blast.cgi) and a SignalP 4.1 Server was used to predict the secretion signal sequences, and the molecular weight and theoretical isoelectric point were calculated using an online tool ExPASy (http://web. expasy.org/compute_pi/). Performance of multiple sequence alignments and the construction of phylogenetic trees were done using the Clustal X package with its default parameters (Livak \& Schmittgen, 2001) and MEGA 5.1 using the neighbour-joining algorithm method (Tamura et al., 2011) with a bootstrap test of 1000 replications, respectively.

\section{Prokaryotic expression and protein purification}

The recombinant fragment of Apserpin-10 was amplified using the pET-Apserpin10-F and pETApserpin10-R primers (Table 1). The recombinant fragment was ligated into cloning vector ( $\mathrm{pMD}$ 19T), cloned and then digested with BamHI and NotI. This digested fragment was then ligated into an expression vector [pET$30 \mathrm{a}(+)]$ and transformed into a prokaryotic expression system $[E$. coli Transetta (DE3)] (AxyGen, Shanghai, China) and sequenced. The bacterial cells were then cultured in Luria-Bertani (LB) media for $4 \mathrm{~h}$; later $0.8 \mathrm{mM}$ isopropyl- $\beta$ - thiogalactopyranoside (IPTG) was added to induce the expression of recombinant protein, and then further cultured for $6 \mathrm{~h}$ and then centrifuged at $5500 \times \mathrm{g}$ for $10 \mathrm{~min}$ and washed twice with PBS (pH 7.4). The pellets (cells) were suspended in a binding buffer $(20 \mathrm{mM}$ Tris-HCl, $500 \mathrm{mM}$ $\mathrm{NaCl}, 5 \mathrm{mM}$ imidazole, $\mathrm{pH}$ 7.9) and disruption of cells was done by sonication in ice. The disrupted cells were then centrifuged for $20 \mathrm{~min}$ at $12,000 \times \mathrm{g}$ and $4^{\circ} \mathrm{C}$, and the QIAexpress ${ }^{\circledR} \mathrm{Ni}-\mathrm{NTA}$ Fast Start Kit (Qiagen, Germany) was used for the purification of recombinant protein according to the manufacturer's instructions. The recombinant protein was subjected to $15 \%$ SDS-PAGE and then western blot analysis was performed using a Mini Trans-Blot Electrophoresis System (Bio-Rad). To quantify the concentration of purified protein a BCA Protein Assay Kit (Novagen, Hilden, Germany) was used.

\section{Antibody preparation}

The Anti-Apserpin-10 polyclonal antibodies were prepared following the protocol of Harlow et al. (1999) by HuaAn (Hangzhou, China). In short, the eluted Apserpin-10 protein $(100 \mu \mathrm{g})$ was injected into New Zealand white rabbits, three times for twoweeks, in order to immunize them. The protein (Apserpin-10) was homogenized in complete Freund's adjuvant before injection. Then a week later the rabbits received a booster injection. Seven days after the booster injection serum was collected from the rabbits and stored in a refrigerator $\left(-80^{\circ} \mathrm{C}\right)$. For the confirmation of protein expression and determination of the molecular

Table 1. Primers used in this study.

\begin{tabular}{lcc}
\hline Primer name & Primer sequence $\left(5^{\prime}-3^{\prime}\right)$ & Purpose \\
\hline $\begin{array}{l}\text { Apserpin10-F } \\
\text { Apserpin10-R }\end{array}$ & ATGCGCCAATTAATTTTGGT & Amplification of ORF \\
\hline pET-Apserpin10-F & TCGCAAAAAAGTTACGTCAAT & Recombinant expression \\
pET-Apserpin10-R & ATTTGCGGCCGCTCGTCAATGACTGTGATTC & \\
\hline qApserpin10-F & GGAAGCAACCGTTCCAAACT & qRT-PCR \\
qApserpin10-R & ATCCCGTGTTCTTGGCAGTA & \\
qAp-18S-F & CGATCCGCCGACGTTACTACA & \\
qAp-18S-R & GTCCGGGCCTGGTGAGATTT & \\
\hline
\end{tabular}

Restriction sites are underlined. 
weight western blotting using His antibody (Qiagen, Germany) was performed.

\section{Transcript analysis}

We performed Quantitative real time polymerase chain reaction (qRT-PCR) to evaluate the expression of Apserpin-10 in A. pernyi. We designed a pair of primers for the Apserpin-10 sequence (qApserpin10-F and qApserpin10-R) and another pair specific for the 18S rRNA (accession number: DQ347469) sequence (internal control) using the Primer 3.0 online primer designing tool (http://bioinfo.ut.ee/primer3-0.4.0/primer3/) (Table 1). $20 \mu \mathrm{L}$ reaction mixture was prepared for each analysis, comprising of $1 \mu \mathrm{L}$ forward primer $(200 \mathrm{mM}), 1 \mu \mathrm{L}$ reverse primer $(200 \mathrm{mM}), 1 \mu \mathrm{L}$ cDNA, $7 \mu \mathrm{L}$ sterile water and $10 \mu \mathrm{L}$ SsoFast EvaGreen SuperMix (Bio-Rad, Hercules, California, USA). Thermal cycling conditions were as follows; $95^{\circ} \mathrm{C}$ for $30 \mathrm{~s}$, followed by 40 cycles of $95^{\circ} \mathrm{C}$ for $5 \mathrm{~s}$ and $60^{\circ} \mathrm{C}$ for $34 \mathrm{~s}$. Each experiment was repeated three times. iCycleriQ TM RT-PCR Detection System (Bio-Rad, Hercules, California, USA) was used to detect amplification. Melting curve analysis was used to further confirm the specificity of the SYBR-Green PCR signal. The quantification of mRNA expression was done using the $2-\Delta \Delta \mathrm{Ct}$ method (Livak \& Schmittgen, 2001).

\section{Apserpin-10 expression in various tissues}

Total RNA from fat body, silk gland, haemocytes, malpighian tubules, mid gut and integument of larvae $\left(5^{\text {th }}\right.$ instar $3^{\text {rd }}$ day) was extracted using TRIzol reagent (Invitrogen). Three larvae constituted a sample, and the biological sampling protocol was repeated three times. Later PrimeScriptTM RT Master Mix (Takara) was used to synthesize the first strand cDNA. qRT-PCR was performed to evaluate the expression profile of Apserpin-10. Expression profile recorded for the mid gut was set to 1 for normalization.

\section{Immunoblot analysis}

To perform the western blot analysis we first extracted total protein from tissues, which was then ground up in liquid nitrogen and dissolved in RIPA lysis buffer (Aidlab Biotech, Beijing, China). The concentration of protein was determined using the bicinchoninic acid assay (BCA) method. $30 \mu \mathrm{g}$ of each protein was subjected to SDS-PAGE. The protein was then transferred from SD-PAGE to a polyvinylidene difluoride (PVDF) membrane (Millipore, Massachusetts, USA) using a Mini Trans-Blot electrophoretic transfer system (Bio-Rad). 5\% non-fat milk diluted with PBST (PBS containing 0.1\% Tween-20) was used for blocking, later after washing with PBST (three times) we incubated the membrane with the prepared Anti-Apserpin-10 polyclonal antibodies (diluted: 400 with 3\% non-fat milk in PBST) for $3 \mathrm{~h}$ at room temperature, then washed it with PBST and incubated it with goat anti-rabbit IgG (Beyotime, Shanghai, China) for $2 \mathrm{~h}$ at room temperature. A HRPDAB Detection Kit (Tiangen, Beijing, China) was used to detect the immunoblot signal.

\section{Developmental profile of Apserpin-10}

We collected samples from different developmental stages to determine the developmental profile of Apserpin-10. Three individuals of each developmental stage were collected as one sample, and the biological sampling protocol was repeated three times. In short total RNA was extracted and first strand cDNA prepared from it. Later qRT-PCR was performed. Apserpin-10 expression in 2nd instar larvae was set to 1 for normalization.

\section{Apserpin-10 expression profile following microbial stress}

Fifth instar larvae of $A$. pernyi (third day) were randomly selected and placed into one of five groups until there were 54 lar- vae in each of the groups. They were then injected with one of four different microorganisms i.e., E. coli (DH5 $\alpha, 1 \times 10^{6}$ cells), A. pernyi nucleopolyhedrovirus (Ap-NPV, $1 \times 10^{6}$ particles), Micrococcus luteus $\left(1 \times 10^{6}\right.$ cells $)$ or Beauveria bassiana $\left(1 \times 10^{6}\right.$ spores), or with phosphate buffer saline (PBS) as a control. $5 \mu \mathrm{L}$ of all microorganisms as well as PBS was injected to the larvae. To perform injections microliter syringes (Gaoge, Beijing, China) were used and vaseline was used to seal the injection site. At different times $(1.5,3,6,12,24$, and $48 \mathrm{~h})$ following the microbial challenge fat bodies and haemolymph were collected. Three larvae constituted a sample and the biological sampling protocol was repeated three times. Total RNA was isolated and then reverse transcribed and qRT-PCR was performed to determine the expression profile of Apserpin-10.

\section{Effect of Apserpin-10 knock-down on transcriptional levels of different Antimicrobial Peptides (AMPs)}

To investigate the effect of Apserpin-10 knock-down on AMPs expression in response to bacterial ( $M$. luteus) invasion, dsRNA for Apserpin-10 and GFP (as the control) were prepared using T7 RiboMAX ${ }^{\mathrm{TM}}$ Express RNAi System (Promega) following the manufacturer's instructions. A. pernyi pupae were injected with 5 $\mu 1,(2 \mathrm{mg} / \mathrm{ml})$ dsRNA (Apserpin-10 or GFP) using microinjection. Twenty four hours after the dsRNA injection, the pupae were injected with $M$. luteus $(5 \mu \mathrm{l}, 0.5 \mu \mathrm{g} / \mu \mathrm{l})$ and then left for another 12 h. Fat body from three pupae were collected as one sample, and the biological sampling protocol was repeated three times. The organisms that were not treated and those challenged with only M. luteus were also used as controls. Later, a qRT-PCR assay was performed to evaluate the difference in transcriptional level of the four different AMPs i.e., Gloverin, Attacin, Moricin and Lebocin, and Apserpin-10 expression was also investigated in both dsApserpin-10 and dsGFP injected groups in order to confirm the knock down of our gene.

\section{Statistical analysis}

One-way analysis of variance (ANOVA) was used to compare data followed by Tukey's test. Data are presented as means \pm standard error (S.E.). The differences were considered significant at $\mathrm{P}<0.05$.

\section{RESULTS}

\section{Bioinformatic analysis of the Apserpin-10 cDNA}

The isolated 1976 bp cDNA fragment of Apserpin-10 consisted of 119 base pairs (bp) $5^{\prime}$-untranslated region (UTR), 300 bp 3' UTR and a 1557 bp open reading frame, encoding a 518 amino acid residue protein. Signal peptide of seventeen amino acids (MRQLILVLFVTVGLSSA) was recorded in the deduced protein sequence (Fig. 1). The protein had a molecular weight of $58.76 \mathrm{kDa}$ and 5.14 isoelectric points. The SMART analysis revealed that Apserpin-10 contains a typical SERPIN domain and is very simi$\operatorname{lar}(69 \%)$ to the serine protease inhibitor 10 of Helicoverpa amigra, which contains the conserved signature of the serpin superfamily. Multiple sequence alignment revealed that the deduced amino acid sequence of Apserpin-10 is very similar to other documented serpin orthologues in Lepidoptera (Fig. 2). A phylogenetic tree was constructed using serpin amino acid residues of invertebrates and vertebrates to determine the evolutionary relationship between Apserpin-10 and other species (Fig. 3). Apserpin-10 was firstly 
1 CGCAGTCGCATGCGTTACTTTITTTTACGTCTGAAACAATATTGTGTTTAATTTATCTATT 61 AGTGCTTCTGTGTTGCGAGCTGTCTGTAATATATATATCTGACGAAAATCGATGACAAA 120 ATGCGCCAATTAATTTTGGTGTTATTTGTGACTGTGGGGCTAAGCAGTGCAAGATGGGTG

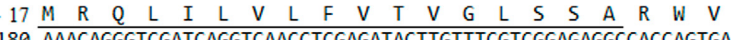
$\begin{array}{llllllllllllllllllll}K & Q & G & R & S & G & Q & P & R & D & T & C & F & V & G & E & A & T & S & E\end{array}$ 240 ATGTCGACTGCTATATTTCAAGGTTATATTGATGATGACAAAAACATTGCATTTTCACCC

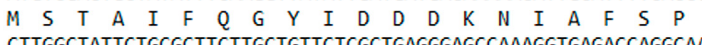
300 CTTGGCTATTCTGCGCTTCTTGCTGTTCTCGCTGAGGGAGCCAAAGGTGAGACCAGGCAA

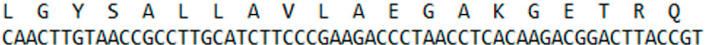
0 L V T A L H L P E D P N L T R R T Y R 20 TACATCATGGAAAGGTTAGCTCACACCCGAGAATATAAATATAACCATCCCGAACTACAG Y I $M$ M E $R$ L L A 480 AATTATTTCTACATATATAAAAACTATACAATCAATGACGATTACAAGAAGATTCTAGAA $\begin{array}{llllllllllllllllllll}N & Y & F & Y & I & Y & K & N & Y & T & I & N & D & D & Y & K & K & I & L & E\end{array}$ 540 GACTATTATATGACAGAAGTGAGGTCTGTTGAGAGATATAACCCAGACCAACAAGCAGAC $\begin{array}{llllllllllllllllllllllllllllllllll}D & Y & Y & M & T & E & V & R & S & V & E & R & Y & N & P & D & 0 & 0 & A & D\end{array}$ 600 AATGATGACGATGAAGGTGAATTCACTATTCACGTGCCAGAGAAGAAGCCAGAAGATAAA

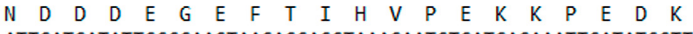
660 ATTGATGATATTCCCGAACTAACACCACCTAAAGAATCTGATGAGAAATTCATATCGTTC $\begin{array}{llllllllllllllllllll}I & D & D & I & P & E & L & T & P & P & K & E & S & D & E & K & F & I & S & F\end{array}$ 720 GCTCTTGAAGACTCTCCAGAGAAAGTAGATGTTACACAAACACAGTATAAACCAGCTAAA

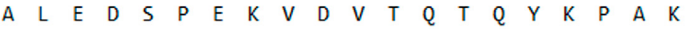
780 AATATTAAGGAGAAAATCAAATTGGTCAATACGTATCCTAAAAGGGAAGAGTCGTTAGAT $N$ N I K K 840 GACGAGGAAACTATGGTCGCTGTTGAAGCTAGAAATCATGTGAGAAACTTCAAGGCTATA $\begin{array}{llllllllllllllllllll}D & E & E & T & M & V & A & V & E & A & R & N & H & V & R & N & F & K & A & I \\ \end{array}$ 900 CATGAAAATCAGGCAACTTCTATGGGGCTTTCAGTGAACAGCGTTGGGAAAGAATCAACA $\begin{array}{llllllllllllllllllll}H & E & N & Q & A & T & S & M & G & L & S & V & N & S & V & G & K & E & S & T\end{array}$ 960 TCGGTGGCAAATTCTCTGATGATAATCTTTAATGGAATGCACTTCCGTGGCTCTTGGAAG S V A N S L M I I F N G M H F $R$ G $S$ W $K$ 1020 CAACCGTTCCAAACTGTTGAACCTGGTATTTTCTACAGGTCTAATTCACAGAAAAAGCAA

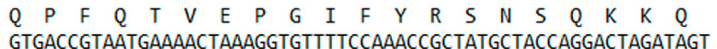

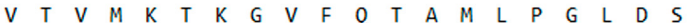
40 GAAGCTATTCGATTACCTTATGCGGGTGATCGTTATGCTCTACTAATTTTACTGCCAAGA E A I R L P Y A G D R Y A L L I L L P R 1200 ACACGTGATGGACTGACCAGACTAATAGCCGATTTACCATCTCTTTCGCTCGATGATATT $\begin{array}{llllllllllllllllllll}T & R & D & G & L & T & R & L & I & A & D & L & P & S & L & S & L & D & D & I\end{array}$ 1260 AATGACAGTTTGCGAAACGAGGAACTGGAAGTCTGTATACCCATATTCAGTGTGGAGACT $\begin{array}{llllllllllllllllllll}N & D & S & L & R & N & E & E & L & E & V & C & I & P & I & F & S & V & E & T\end{array}$ 1320 ACAACTCGACCAATTGGTGCTTTGGCGAAGCTTGGAATTAACAAGATTTTCAACCGTAAT

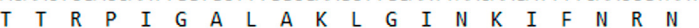
1380 GCGGAACTGTCCGGAGTGTCTTCGGACGAAGGGCTCTTCGTCAAAGAATTGGTTCAGTTC $\begin{array}{lllllllllllllllllllll}A & E & L & S & G & V & S & S & D & E & G & L & F & V & K & E & L & V & Q & F & 423\end{array}$ 1440 GTGTCTGTTCGGGTAGATAACACTGAATCATCGGTTTCTGAGATATCAGCTGGCAACCCT

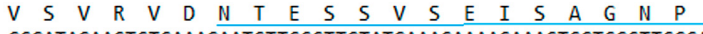
1500 GCGATAGAACTCTCAAAGAATCTTCCCTTGTATGAAACAAAACAAACTCGTCGCTTCGCA \begin{tabular}{llllllllllllllllllll}
$A$ & $I$ & $E$ & $L$ & $S$ & $K$ & $N$ & $L$ & $P$ & $L$ & $Y$ & $E$ & $T$ & $K$ & $Q$ & $T$ & $R$ & $R$ & $F$ & $A$ \\
\hline
\end{tabular} 1560 ATCAAC \begin{tabular}{lllllllllllllllllllll}
$I$ & $N$ & $R$ & $P$ & $F$ & $V$ & $F$ & $Y$ & $I$ & $V$ & $D$ & $T$ & $L$ & $D$ & $D$ & $L$ & $V$ & $V$ & $V$ & $A$ \\
\hline
\end{tabular} 1620 GGAAAGGTCACCAACCCTGAACAACCTACGCCGTTTGAATCACAGTCATTGACGTAACTT $\begin{array}{lllllllllllllll}G & K & V & T & N & P & E & Q & P & T & P & F & E & S & Q\end{array}$ TI40 TITCCATTTTTGAAAATCTACATTCTTTATTACCCTGTCTATTATTTAATTATTTTCGG 1800 TAATTGAGAACTGGTCCTATCTATTTTCATTATATACTCTCAATATTATTTGTTTATTGC 1920 ACACAGCTTAGTATTTAGTTAGTTTAAGTTATTATTTATTTTTTTGTATGTAACGTG

Fig. 1. Nucleotide and deduced amino acid sequence of Apserpin-10 of $A$. pernyi. The cDNA nucleotide sequence is shown above the deduced amino acid sequence and the one-letter codes are aligned with the second nucleotide of each codon. The nucleotide sequence is numbered on the left and the amino acid sequence on the right. Amino acid residues in the mature protein are assigned positive numbers, and those in the signal peptide are assigned negative numbers (black underlined). The termination codon TAA is marked with an asterisk. The predicted $\mathrm{RCL}$ is underlined in blue.

Fig. 2. Alignment of the deduced amino acid sequence of Apserpin-10 with homologous proteins. The deduced amino acid sequence of Apserpin-10 are aligned with the serpins from Helicoverpa armigera (GenBank accession number: APM86798.1), Amyelois transitella (XP_013186212.1), Danaus plexippus (EHJ73924.1), Pararge aegeria (JAA89165.1), Heliconius melpomene (AOG75389.1), Papilio machaon (KPJ15998.1), Bombyx mori (NP_001139703.1), Loxodonta africana (XP_003416302.2), Hipposideros armiger (XP 019499453.1), Miniopterus natalensis (XP_016071207.1), Xenopus tropicalis (NP_001015754.1), Culex quinquefasciatus (XP 001867224.1), Nilaparvata lugens (AGK40929.1), Ixodes scapularis (XP_002399564.1) and Carlito syrichta (XP 008065456.1).
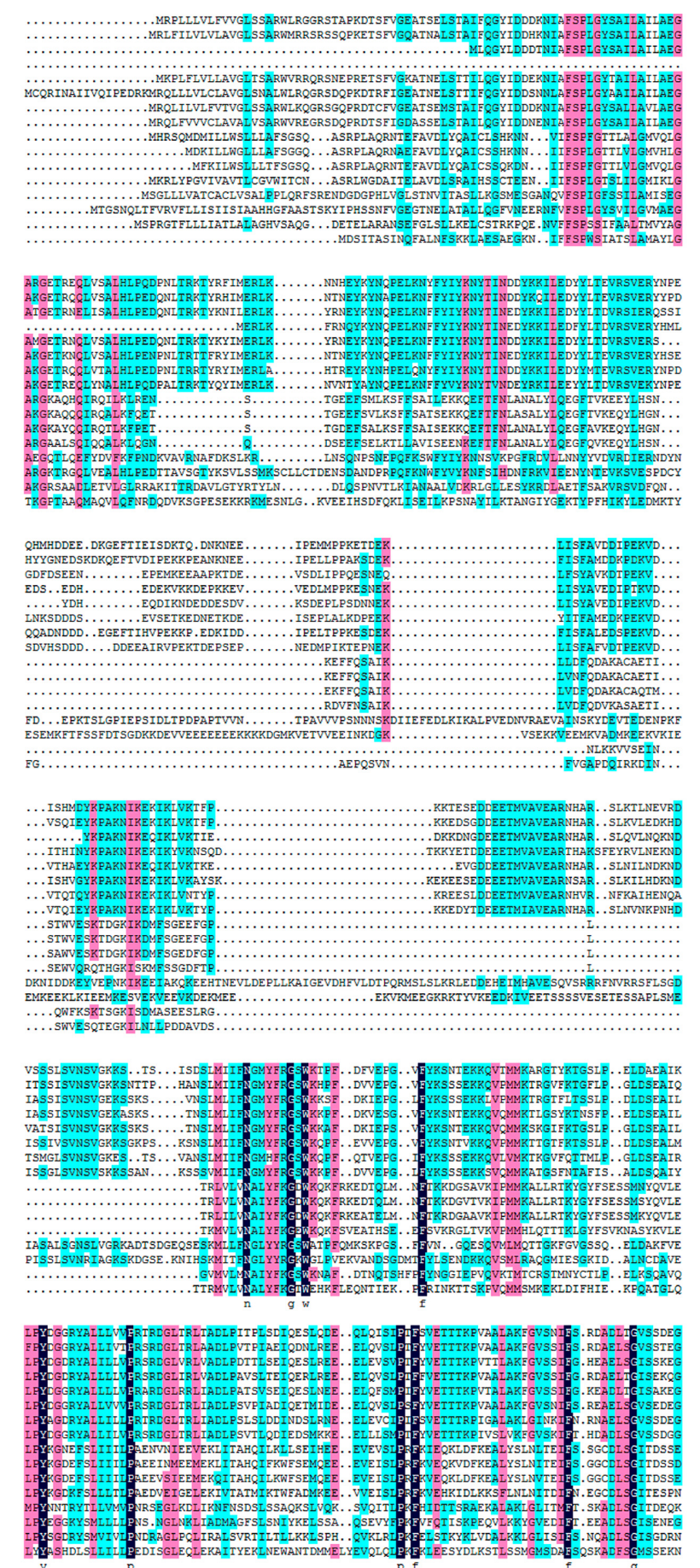

431
436
376
326
414
447
431
431
330
325
324
331
496
487
333
330
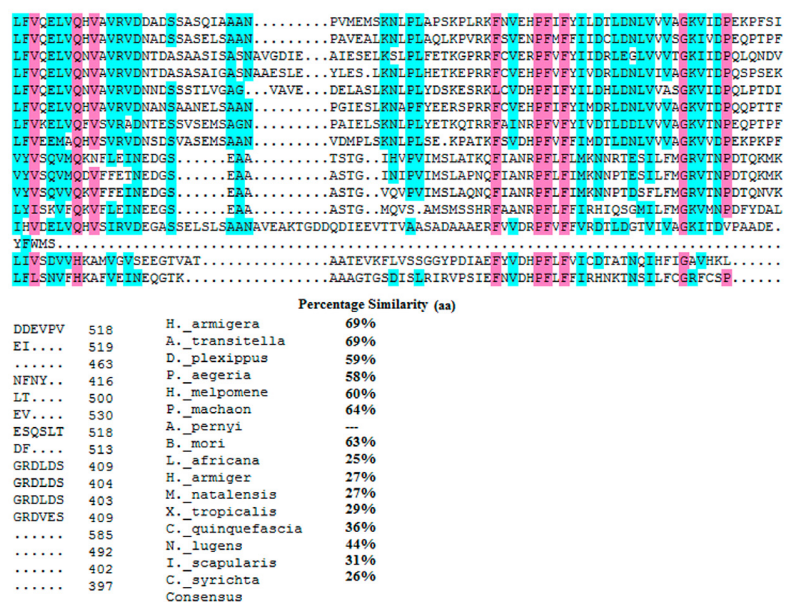


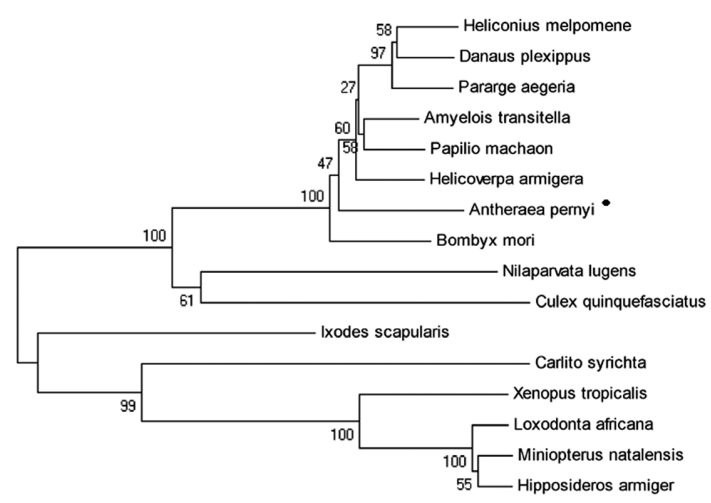

0.1

Fig. 3. Phylogenetic analysis using Apsepin-10 and other serpins. The phylogenetic tree was constructed using the neighbour-joining algorithm and the bootstrap values (1000 repetitions) of the branches are indicated. The sequences from Helicoverpa armigera (GenBank accession number: APM86798.1), Amyelois transitella (XP 013186212.1), Danaus plexippus (EHJ7 3924.1), Pararge aegeria (JAA89165.1), Heliconius melpomene (AOG75389.1), Papilio machaon (KPJ15998.1), Bombyx mori (NP_001139703.1), Loxodonta africana (XP_003416302.2), Hipposideros armiger (XP_019499453.1), Miniopterus natalensis (XP_016071207.1), Xenopus tropicalis (NP 001015754.1), Culex quinquefasciatus (XP_001867224.1), Nilaparvata lugens (AGK40929.1), Ixodes scapularis (XP 002399564.1) and Carlito syrichta (XP_008065456.1) were used to construct the phylogenetic tree.

clustered with other Lepidoptera to form a separate clade and then with vertebrate serpin proteins.

\section{Expression of recombinant Apserpin-10, antibody synthesis and immunoblot analysis}

The Apserpin-10 protein was expressed using a prokaryotic expression system (E. coli). The recombinant protein expression was confirmed using SDS-PAGE analysis (Fig. 4a). The Apserpin-10 $\times$ His fusion protein was purified by using affinity chromatography and the protein single band of predicted molecular weight $(58.76 \mathrm{kDa})$ was detected using SDS page (Fig. 4b). Western blotting analysis of the extracted protein using an anti-His-tag antibody confirmed the presence of recombinant Apserpin-10 (Fig. 4c). The purified recombinant Apserpin-10 protein was then used to prepare rabbit anti-Apserpin-10 antibodies.

\section{Expression of Apserpin-10 in larval tissues}

The level of expression of Apserpin-10 mRNA in haemocytes, fat bodies, mid gut, silk glands, epidermis and Malpighian tubules was determined using qRT-PCR. $A p$ serpin-10 was transcribed in all the tissues examined; however, its expression in fat body and haemocytes was higher than in other tissues (Fig. 5a). Western blotting analysis of proteins extracted from these tissues revealed that Apserpin-10 was detectable in all the tissues examined (Fig. 5b).

\section{Developmental profile of Apserpin-10 expression}

To evaluate the expression of the Apserpin-10 gene in different developmental stages of $A$. pernyi, we extracted total RNA from eggs, larvae (1st to 5 th instar), pupae and adult stages and then reverse transcribed to synthesize cDNA, later qRT-PCR was performed to determine the relative mRNA expression. As seen in Fig. 6, Apserpin-10

\section{$A$}

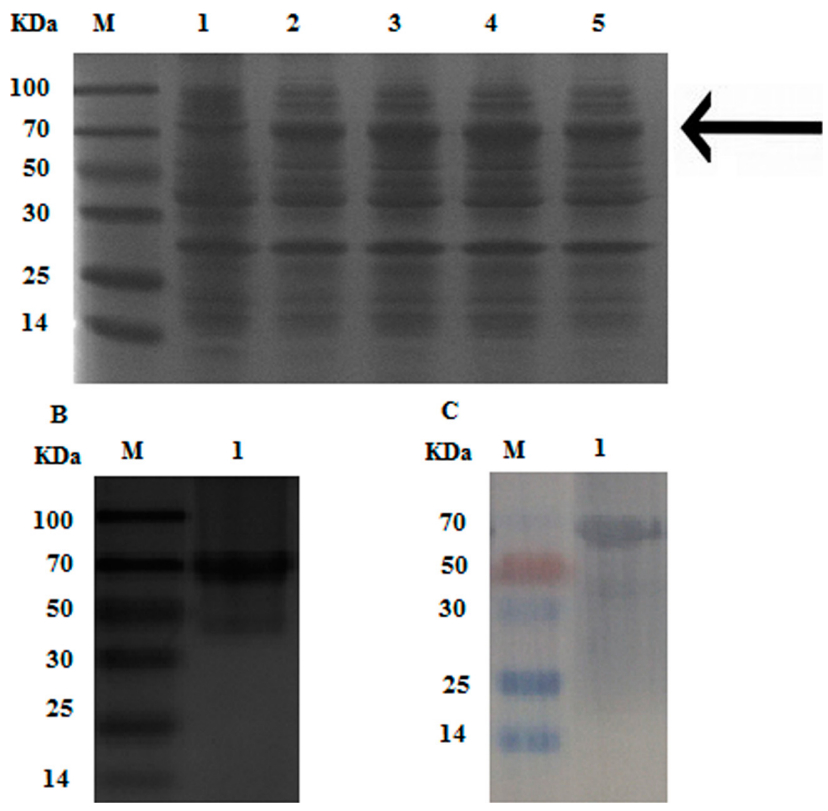

Fig. 4. Expression and purification of recombinant Apserpin-10. (A) Analysis of recombinant Apserpin-10 protein on $12 \%$ SDSPAGE gel. (B) Extracted Apserpin-10 protein. (C) Western blotting analysis of recombinant proteins using anti-His-tag antibodies. Bacterial proteins were collected after $4 \mathrm{~h}$ of induction with different IPTG concentrations. M, molecular weight marker; Lane 1, non-induced $E$. coli (pET-Apserpin-10); Lane 2, induction of E. coli (pET-Apserpin-10) using 0.6 mM IPTG; Lane 3, induction of E. coli (pET-Apserpin-10) using 0.8 mM IPTG; Lane 4, induction of E. coli (pET-Apserpin-10) using 1 mM IPTG; Lane 5, induction of $E$. coli (pET-Apserpin-10) using 1.5 mM IPTG.

mRNA was expressed at high levels in the 5th instar larvae followed by pupae, 3rd, 4th and 1st instar larvae, and the lowest transcript expression was recorded in adults and eggs.
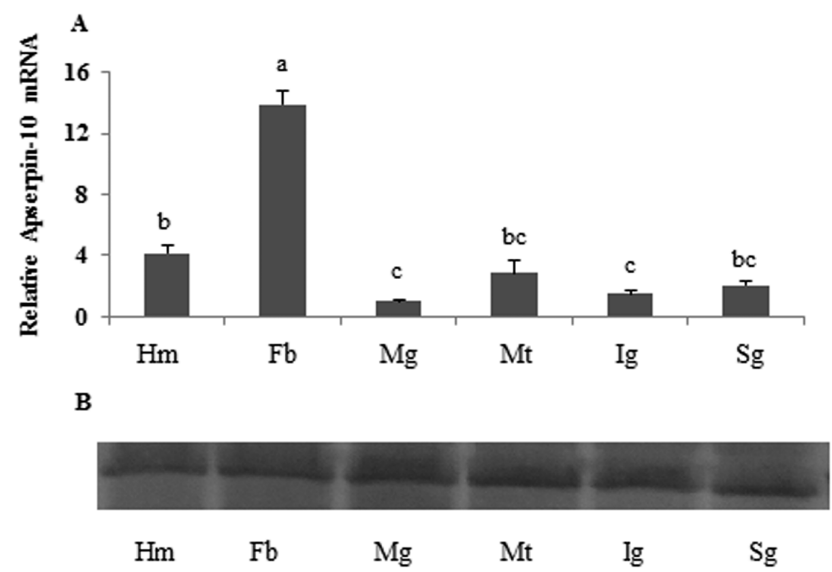

Fig. 5. Distribution of Apserpin-10 in the tissues of 5 th instar larvae of A. pernyi. (A) Analysis of Apserpin-10 transcript expression in different tissues of larvae using qRT-PCR. The Apserpin-10 transcript level in the mid gut was used as the calibrator. Bars represent means \pm S.E. $(n=3)$. Bars labelled with different letters are significantly different (one-way ANOVA followed by Tukey's test, $P<0.05)(B)$ Analysis of Apserpin-10 protein expression in different tissues by western blotting. Apserpin-10 was detected using anti-Apserpin-10 rabbit polyclonal antibodies. $\mathrm{Mg}-$ mid gut, $\mathrm{Hm}-$ haemocyte, $\mathrm{Fb}$ - fat body, Mt - Malpighian tubule, Im - integument, $\mathrm{Sg}$ - silk gland. 


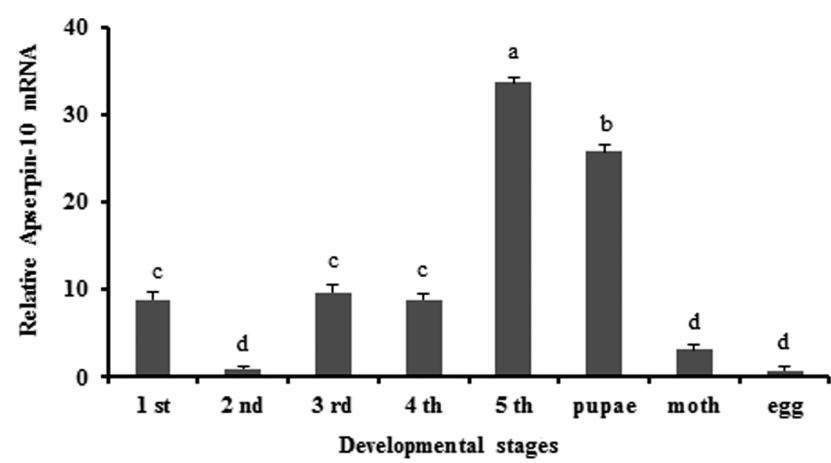

Fig. 6. Expression of Apserpin-10 in the different developmental stages of $A$. pernyi. Analysis of Apserpin-10 transcript expression in different developmental stages of larvae using qRT-PCR. The Apserpin-10 transcript level in the 2nd instar was used as the calibrator. Bars represent means \pm S.E. $(n=3)$. Bars labelled with different letters are significantly different (one-way ANOVA followed by Tukey's test, $P<0.05$ ).

\section{Apserpin-10 expression profile under microbial stress conditions}

To understand whether Apserpin-10 is involved in or influences the immune process of $A$. pernyi under biotic stress, we injected fungal, bacterial and viral pathogens into 5th instar larvae (3rd day). Total RNA was isolated from the fat body and haemocytes at different time intervals from 1.5 to $48 \mathrm{~h}$, and was then investigated using qRT-PCR. When $A$. pernyi larvae were treated with the different pathogens, the level of Apserpin-10 in the fat body and haemocytes varied considerably and the tissue mRNA expression varied with the kind of pathogen. Overall viral, fungal and bacterial pathogens greatly enhanced the expression of Apserpin-10; however the expression level and time of maximum expression varied. In fat body, NPV produced three expression peaks at 12, 24 and $48 \mathrm{~h}$, and the maximum expression in response to $B$. bassiana was recorded after $48 \mathrm{~h}$. Meanwhile, two expression peaks were recorded (at 24 and $48 \mathrm{~h}$ ) following challenge from E. coli and the highest transcript expression was recorded after 48 $\mathrm{h}$ followed by 12 and $6 \mathrm{~h}$ after the challenge from $M$. luteus (Fig. 7). Approximately similar trends were recorded

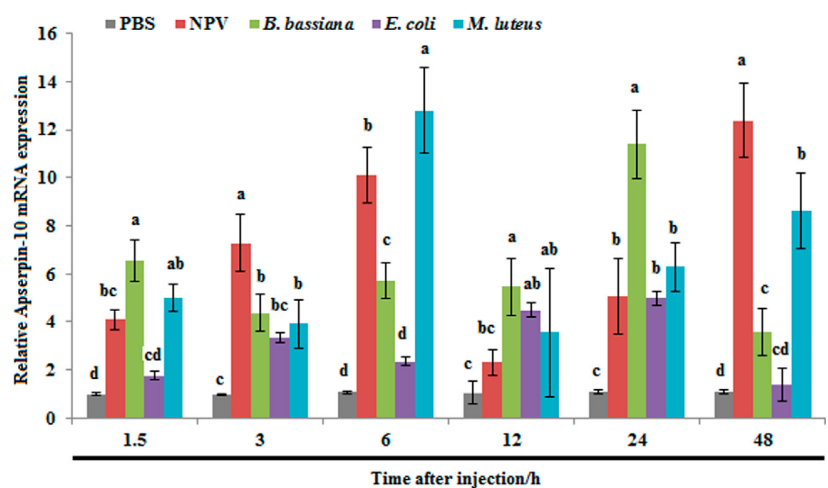

Fig. 7. Expression of Apserpin-10 in haemocytes after microbial challenge in the fifth instar larvae of $A$. pernyi $1.5 \mathrm{~h}$ to $48 \mathrm{~h}$ following challenges from nucleopolyhedrovirus (NPV), B. bassiana, $E$. coli and M. luteus. PBS was injected as a control. Bars represent means \pm S.E. $(n=3)$. Bars labelled with different letters are significantly different (one-way ANOVA followed by Tukey's test, $\mathrm{P}<$ 0.05).

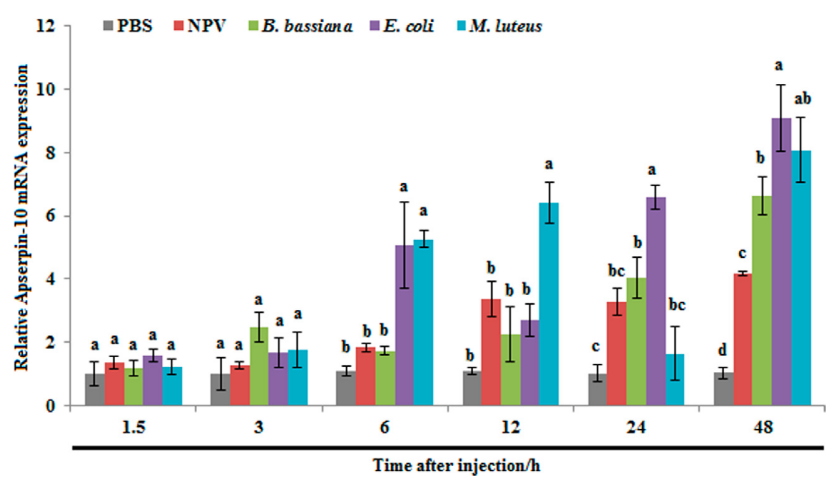

Fig. 8. Expression of Apserpin-10 in fat body after microbial challenge in the fifth instar larvae of $A$. pernyi $1.5 \mathrm{~h}$ to $48 \mathrm{~h}$ following challenges from nucleopolyhedrovirus (NPV), B. bassiana, $E$. coli and M. luteus. PBS was injected as a control. Bars represent means \pm S.E. $(n=3)$. Bars labelled with different letters are significantly different (one-way ANOVA followed by Tukey's test, P < 0.05).

in haemocytes after treatment with the pathogens. The expression in the haemocytes varied more depending on the pathogen and time interval than that recorded for the fat body. The maximum expression level in response to NPV was recorded at $48 \mathrm{~h}$, whereas it was $24 \mathrm{~h}$ for the response to $B$. bassiana. The expression level of Apserpin-10 was much higher in response to $E$. coli and M. luteus: however, the maximum level recorded in response to $E$. coli occurred at $24 \mathrm{~h}$ and at $6 \mathrm{~h}$ in response to M. luteus (Fig. 8).

\section{Involvement of Apserpin-10 in transcriptional level of AMPs}

To explore the effect of Apserpin-10 on humoral immune responses in $A$. pernyi, we evaluated the transcriptional level of AMPs in response to bacterial (M. luteus)

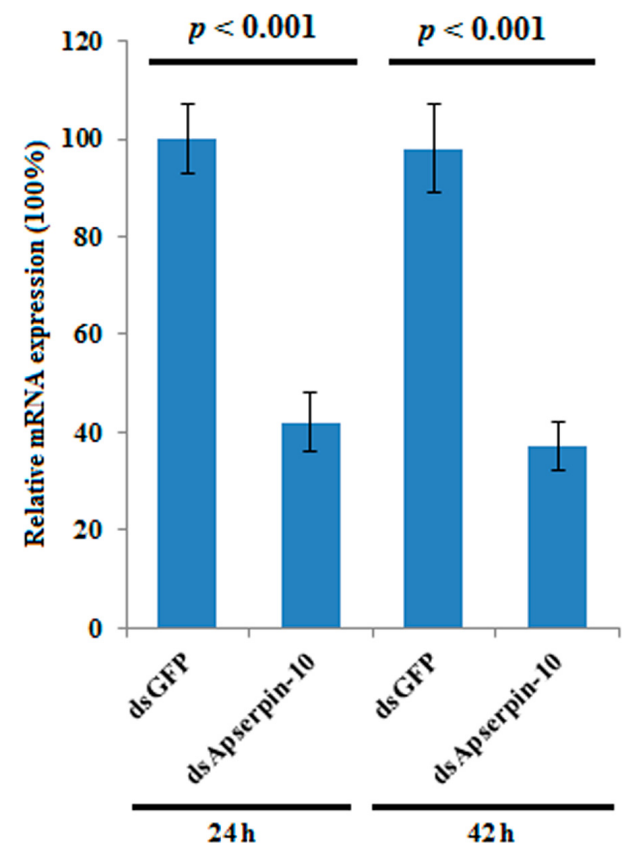

Fig. 9. RNAi efficiency estimation of Apserpin-10 Relative levels of mRNA expression of Apserpin-10 in ds GFP treated fat body and Apserpin-10 dsRNA treated fat body after 24 and $42 \mathrm{~h}$ using qRT PCR. Bars represent means \pm S.E. $(n=3)$. Bars labelled with different letters are significantly different (one-way ANOVA followed by Tukey's test, $\mathrm{P}<0.05$ ). 

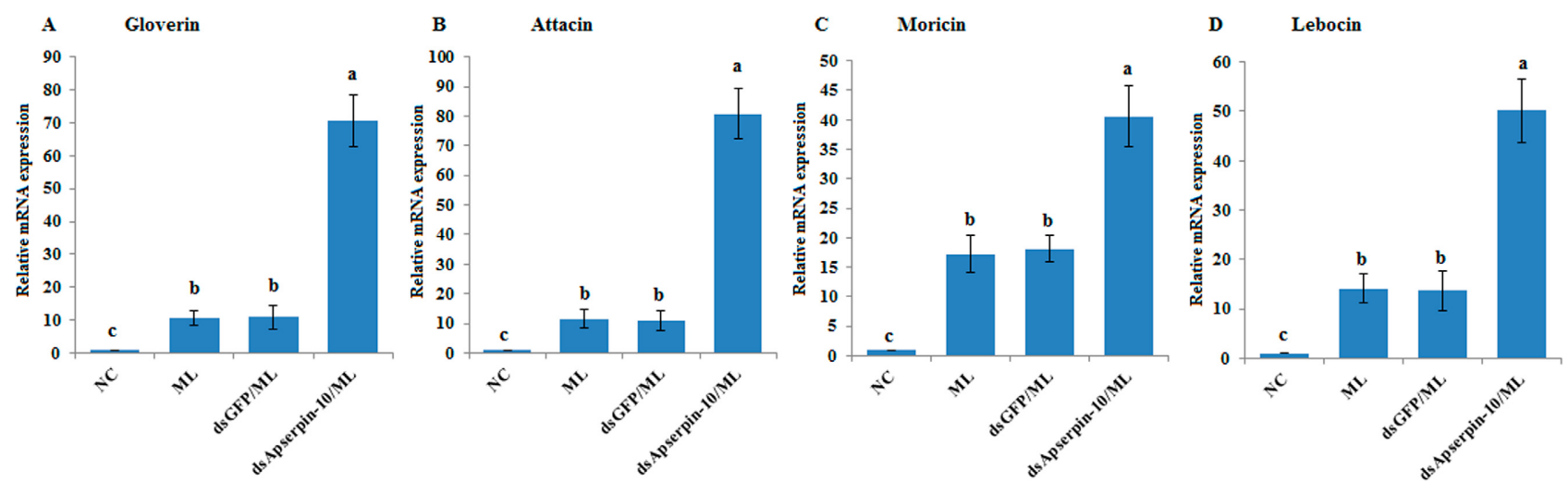

Fig. 10. Expression levels of antimicrobial peptides in response to Apserpin-10 knock-down. Relative levels of mRNA expression of particular antimicrobial peptides, including gloverin (A), attacin (B), moricin (C) and lebocin (D). NC - untreated sample; $M L-M$. luteus treated; dsGFP/ML - both dsGFP and M. luteus treated; dsApserpin-10/ML - dsApserpin-10 and M. luteus treated. Bars represent means \pm S.E. $(n=3)$. Bars labelled with different letters are significantly different (one-way ANOVA followed by Tukey's test, $P<0.05$ ).

challenge after the depletion of Apserpin-10. Prior to this analysis the AMPs expression, the interference efficiency of ds-RNA injection were evaluated using qRT-PCR. As shown in Fig. 9, the mRNA level of Apserpin-10 was significantly suppressed $(\sim 60 \%)$ after $24 \mathrm{~h}$ and $42 \mathrm{~h}$ by injecting dsApserpin-10 compared to dsGFP (Control group).

The relative mRNA expression of AMPs (Gloverin, Attacin, Moricin and Lebocin) was significantly enhanced in the dsApserpin-10/M. luteus treated sample compared to $\mathrm{dsGFP} / M$. luteus and only in $M$. luteus challenged samples. Whereas, the AMPs expression did not differ significantly in the M. luteus injected and dsGFP/M. luteus treated samples (Fig. 10A-D).

\section{DISCUSSION}

Here we isolated and characterized serpin-10 gene from A. pernyi. The Apserpin-10 sequence was very similar to serpin 10 of $H$. armigera and A. transitella. Further, a typical serpin domain and the RCL were also present in the sequence. Based on these observations, we assumed that the gene studied is an orthologue of serpin-10. Both in invertebrates and vertebrates, many serpins have been identified and characterized as factors involved in the regulation of immune responses (Potempa et al., 1994; Jiang \& Kanost, 1997; Law et al., 2006).

The pattern in the spatial pattern of Apserpin-10 in tissues examined at different developmental stages could provide valuable clues about its biological role in $A$. pernyi. In this study, Apserpin-10 was expressed in all the tissues tested, although the expression levels varied between tissues. The Apserpin-10 transcript levels were highest in fat body and haemocytes, and lowest in integument and mid gut (Fig. 5). This expression profile of Apserpin-10 is consistent with that of $M$. configurata serpin-1d (Hegedus et al., 2008), Choristoneura fumiferana serpin-1 (Zheng et al., 2009) and A. pernyi serpin-3 and 14 (Kausar et al., 2017; Wang et al., 2017). It is generally believed that the insect's fat body has an important role in homeostasis and the immune response (Feng et al., 2011) and the high expression of Apserpin-10 mRNA in fat body and haemocytes was probably an immune response against microbial pathogens. Apserpin-10 mRNA was ubiquitously expressed in all developmental stages, although the levels of expression differed. The maximum transcript level of Apserpin-10 was recorded in $5^{\text {th }}$ instar larvae followed by pupae and the lowest expression in $2^{\text {nd }}$ instar larvae, adults and eggs. These results are consistent with developmental profile of serpin 1 in A. pernyi (Yu et al., 2017). Similarly, Han et al. (2014) report low levels of the mRNA of three serpins (serpin 2, 4 and 5) in $2^{\text {nd }}$ instar larvae of Plutella xylostella compared to other stages. Contrary to the present study, Pan et al. (2009) report a low level of expression of serpin 2 in first instar larvae and higher and increasing levels in other life stages of $B$. mori. The literature generally indicates that different serpins are ubiquitously expressed at all developmental stages, however their level of expression vary in different life stages. The variable expression pattern of different serpins may be associated with physiological requirements of the different life stages of an organism.

Previous studies indicate that serpins are involved in many immune processes such as serine protease mediated defence against microbial infection and impairment of tissues (Kanost, 1999; Jiang, 2008). Haemocytes and fat body are considered to be important tissues in the regulation of immune processes (Feng et al., 2011) in insects as they are the main producers of antimicrobial peptides, which they secrete into the haemolymph to combat invading pathogens (Tanaka \& Yamakawa, 2011). Therefore, in the present study, we analyzed the expression profile of Apserpin-10 in haemocytes and fat body after an immunological challenge (bacterial, viral and fungal). The Apserpin-10 transcript was found to be significantly up-regulated at different times after microbial infection. Several serpins in $A$. pernyi such as serpin-1 (Yu et al., 2017), serpin-3 (Wang et al., 2017) and serpin-14 (Kausar et al., 2017) are reported to be up-regulated after pathogen challenge. Furthermore, serpin-15 mRNA expression increases in the fat body of $B$. mori following bacterial, fungal and viral injections (Liu et al., 2015). This kind of trend in the expression is also reported for most insect serpins (Danielli et al., 2003; Zheng et al., 2009; Zou et al., 2009; Gulley et al., 2013). All these authors suggest that these serpins regulate specific immune 
reactions in insects and it is likely that Apserpin-10 has a crucial role in the regulation of the immune processes in A. pernyi.

Antimicrobial peptides (AMPs) play a crucial role in the humoral defence of arthropods. In insects, they are mainly synthesized by IMD and Toll signalling pathways. In the Toll pathway, a serine proteinase cascade similar to the prophenol oxidase activation system activates spatzle, the ligand of the trans-membrane Toll receptor (Kim et al., 2008; Roh et al., 2009; Aymeric et al., 2010). Further, such proteinase cascades are controlled by serpins. In the present study, we investigated the effect of Apserpin-10 knockdown on the expression of AMPs. The mRNA level of all the AMPs tested increased significantly following Apserpin-10 depletion, indicating it may negatively regulate the production of AMPs in A. pernyi. Similarly, in Manduca sexta, hemolymph proteinase 6 stimulates the activation of the Toll pathway and the inhibition of this proteinase by serpin- 5 appears to negatively regulate the synthesis of antimicrobial peptides (An et al., 2009). In Drosophila melanogaster Serpin-43 C (Necrotic) and serpin-1 regulate the Toll signalling pathway (Levashina, 1999; Green et al., 2000; Fullaondo et al., 2011). In addition, Liu et al. (2015) report a significant suppression of the expression of AMPs in $B$. mori after injection of recombinant serpin- 15 protein. Our data indicate that Apserpin-10 may play a role in the regulation of the Toll pathway and may be involved in the innate immune responses of $A$. pernyi.

We conclude that Apserpin-10 is involved in the immune responses against pathogens. Although we investigated the level of expression of Apserpin-10 in different tissues and developmental stages of $A$. pernyi after microbial stress, the mechanisms involved in the interaction between Apserpin-10 and its effectors and its signalling pathways remain unclear. More studies are needed to evaluate these molecular and signalling mechanisms in order to provide a clearer understanding of the interactions between Apserpin-10 and its effectors.

ACKNOWLEDGEMENTS. This work was supported by the Earmarked Fund for Modern Agro-industry Technology Research System (grant number CARS-22-SYZ10), the National Natural Science Foundation of China (grant numbers 31301715, 31472147, 31402018), the Anhui Provincial Natural Science Foundation of China (grant number 1308085QC60), the sericulture Biotechnology Innovation Team 410 (grant number 2013xkdt-05) and Ph.D Programs in Biochemistry and Molecular Biology (grant number xk2013042).

\section{REFERENCES}

Abraham E.G., Pinto S.B., Ghosh A., Vanlandingham D.L., Budd A., Higgs S., Kafatos F.C., Jacobs L.M. \& Michel K. 2005: An immune-responsive serpin, SRPN6, mediates mosquito defence against malaria parasites. - Proc. Natn. Acad. Sci. U.S.A. 102: 16327-6332.

An C., Ishibashi J., Ragan E.J., Jiang H. \& Kanost M.R. 2009: Functions of Manduca sexta hemolymph proteinases HP6 and HP8 in two innate immune pathways. - J. Biol. Chem. 284: 19716-19726.
Aymeric J.L., Alain G. \& Bernard D. 2010: Imd pathway is involved in the interaction of Drosophila melanogaster with the entomopathogenic bacteria, Xenorhabdus nematophila and Photorhabdus luminescens. - Mol. Immunol. 47: 2342-2348.

Bulet P., Hetru C., Dimarce J.L. \& Hoffmann D. 1999: Antimicrobial peptides in insects: structure and function. - Dev. Comp. Immunol. 23: 329-344.

Danielli A., Kafatos F.C. \& Loukeris T.G. 2003: Cloning and characterization of four Anopheles gambiae serpin isoforms, differentially induced in the midgut by Plasmodium berghei invasion. - J. Biol. Chem. 278: 4184-4193.

De Gregorio E., Han S.J., Lee W.J., Baek M.J., Osaki T., Kawabata S., Lee B.L., Iwanaga S., Lemaitre B. \& Brey P.T. 2002: An immune-responsive serpin regulates the melanization cascade in Drosophila. - Dev. Cell 3: 581-592.

Feng C., Huang J., Song Q., Stanley D., Lu W., Zhang Y. \& HuAng Y. 2011: Parasitization by Macrocentrus cingulum (Hymenoptera: Braconidae) influences expression of prophenoloxidase in Asian corn borer Ostrinia furnacalis. - Arch. Insect Biochem. Physiol. 77: 99-117.

Fullaondo A., Garcia-Sanchez S., Sanz-Parra A., Recio E., Lee S.Y. \& GuBb D. 2011: Spn1 regulates the GNBP3-dependent Toll signaling pathway in Drosophila melanogaster. - Mol. Cell Biol. 31: 2960-2972.

Gooptu B. \& Lomas D.A. 2009: Conformational pathology of the serpins: themes, variations, and therapeutic strategies. Annu. Rev. Biochem. 78: 147-176.

Green C., Levashina E., McKimmie C., Dafforn T., Reichhart J.M. \& GubB D. 2000: The necrotic gene in Drosophila corresponds to one of a cluster of three serpin transcripts mapping at 43A1.2. - Genetics 156: 1117-1127.

Gubb D., Robertson A., Dafforn T., Troxler L. \& Reichhart J.M. 2007: Drosophila serpins: Regulatory cascades in innate immunity and morphogenesis. In Silverman G.A. \& Lomas D.A (eds): Molecular and Cellular Aspects of the Serpinopathies and Disorders in Serpin Activity. World Scientific Publishing, Singapore, pp. 207-227.

Gulley M.M., Zhang X. \& Michel K. 2013: The roles of serpins in mosquito immunology and physiology. - J. Insect Physiol. 59: $138-147$.

Han P., Fan J., Liu Y., Cuthbertson A.G.S., Yan S., Qiu B.L. \& REN S. 2014: RNAi-mediated knockdown of serine protease inhibitor genes increases the mortality of Plutella xylostella challenged by destruxin A. - PLOS ONE 9: e97863, 16 pp.

Harlow E. \& Lane D. 1999: Using Antibodies: A Laboratory Manual. Cold Spring Harbor Laboratory Press, New York, 726 pp.

Hegedus D.D., Erlandson M., Baldwin D., Hou X. \& ChaMANKHAH M. 2008: Differential expansion and evolution of the exon family encoding the serpin-1 reactive centre loop has resulted in divergent serpin repertoires among the Lepidoptera. - Gene 418: 15-21.

Irving J.A., PIKe R.N., Lesk A.M. \& Whisstock J.C. 2000: Phylogeny of the serpin superfamily: implications of patterns of amino acid conservation for structure and function. - Genome Res. 10: 1845-1864.

JIANG H. 2008: The biochemical basis of antimicrobial responses in Manduca sexta. - Insect Sci. 15: 53-66.

JiANG H. \& KANOST M.R. 1997: Characterization and functional analysis of 12 naturally occurring reactive site variants of serpin-1 from Manduca sexta. - J. Biol. Chem. 272: 1082-1087.

KANOST M.R. 1999: Serine proteinase inhibitors in arthropod immunity. - Dev. Comp. Immunol. 23: 291-301.

Kausar S., Abbas M.N., Qian C., Zhu B.J., Sun Y., Sun Y.X., Wang L., Wei G., Maqsood I. \& Liu C.L. 2017: Serpin-14 neg- 
atively regulates prophenoloxidase activation and expression of antimicrobial peptides in Chinese oak silkworm Antheraea pernyi. - Dev. Comp. Immunol. 76: 45-55.

Kim C.H., Kim S.J., Kan H., Kwon H.M., Roh K.B., Jiang R., Yang Y., Park J.W., Lee H.H., Ha N.C., Kang H.J., Nonaka M., Soderha K. \& Lee B.L. 2008: A three step proteolytic cascade mediates the activation of the peptidoglycan-induced Toll pathway in an insect. - J. Biol. Chem. 283: 7599-7607.

Law R.H., Zhang Q., McGowan S., Buckle A.M., Silverman G.A., Wong W., Rosado C.J., Langendorf C.G., Pike R.N., Bird P.I. \& Whisstock J.C. 2006: An overview of the serpin superfamily. - Genome Biol. 7: 216, 11 pp.

Levashina E.A. 1999: Constitutive activation of toll-mediated antifungal defense in serpin-deficient Drosophila. - Science 285: 1917-1919.

Li W., Terenius O., Hirai M., Nilsson A.S. \& Faye I. 2005: Cloning, expression and phylogenetic analysis of hemolin, from the Chinese oak silkmoth, Antheraea pernyi. - Dev. Comp. Immunol. 29: 853-864.

Liu D.R., Wang L., Yang L., Qian C., Wei G.Q., Dai L.S., Li J., Zнu B.J. \& Liu C.L. 2015: Serpin-15 from Bombyx mori inhibits prophenoloxidase activation and expression of antimicrobial peptides. - Dev. Comp. Immunol. 51: 22-28.

LivaK K.J. \& SCHMitTGEN T.D. 2001: Analysis of relative gene expression data using real-time quantitative PCR and the 2(-Delta Delta C(T)) method. - Methods 25: 402-408.

Lu W.X., Yue D., Hai Z.J., Dainua W., Yi Z.M., Fu W.C. \& Rong Z. 2015: Cloning, expression, and characterization of prophenoloxidase from Antheraea pernyi. - Arch. Insect Biochem. Physiol. 88: 45-63.

Pan Y., Xia H., Lü P., Chen K., Yao Q., Chen H., Gao L., He Y. \& WANG L. 2009: Molecular cloning, expression and characterization of Bmserpin-2 gene from Bombyx mori. - Acta Biochim. Pol. 56: 671-677.

Potempa J., Korzus E. \& Travis J. 1994: The serpin superfamily of proteinase inhibitors: structure, function, and regulation. J. Biol. Chem. 269: 15957-15960.

Rawlings N.D., Tolle D.P. \& Barrett A.J. 2004: MEROPS: the peptidase database. - Nucl. Acids Res. 32: 160-164.

ReIchHart J.M. 2005: Tip of another iceberg: Drosophila serpins. — Trends Cell Biol. 15: 659-665.

Roh K.B., Kim C.H., Lee H., Kwon H.M., Park J.W., Ryu J.H., Kurokawa K., Ha N.C., Lee W.J., Lemaitre B., Soderhall K. \& LEE B.L. 2009: Proteolytic cascade for the activation of the insect toll pathway induced by the fungal cell wall component. - J. Biol. Chem. 284: 19474-19481.

Schleef R.R. \& Chuang T.L. 2000: Protease inhibitor 10 inhibits tumor necrosis factor alpha-induced cell death. Evidence for the formation of intracellular high $\mathrm{M}(\mathrm{r})$ protease inhibitor 10-containing complexes. - J. Biol. Chem. 275: 26385-26389.

Silverman G.A., Bird P.I., Carrell R.W., Church F.C., Coughlin P.B., Gettins P.G., Irving J.A., Lomas D.A., LuKe C.J., Moyer R.W., Pemberton P.A., Remold-O’Donnell E., Salvesen G.S., Travis J. \& Whisstock J.C. 2001: The serpins are an expanding superfamily of structurally similar but functionally diverse proteins. Evolution, mechanism of inhibition, novel functions, and a revised nomenclature. - J. Biol. Chem. 276: 33293-33296.

SuWANChaichinda C. \& Kanost M.R. 2009: The serpin gene family in Anopheles gambiae. - Gene 442: 47-54.

Tamura K., Peterson D., Peterson N., Stecher G., Nei M. \& KuMAR S. 2011: MEGA5: Molecular evolutionary genetics analysis using maximum likelihood, evolutionary distance, and maximum parsimony methods. - Mol. Biol. Evol. 28: 2731-2739.

TANaKa H. \& Yamakawa M. 2011: Regulation of the innate immune responses in the silkworm, Bombyx mori. - Invert. Surviv. J. 8: $59-69$.

Wang X., Zhang J., Chen Y., Ma Y., Zou W., Ding G., Li W., Zhao M., Wu C. \& Zhang R. 2013: A novel pattern recognition protein of the Chinese oak silkmoth, Antheraea pernyi, is involved in the pro-PO activating system. - BMB Rep. 46: 358-363.

Wang X., Wang K., He Y., Lu X., Wen D., Wu C., Zhang J. \& ZHANG R. 2017: The functions of serpin-3, a negative-regulator involved in prophenoloxidase activation and antimicrobial peptides expression of Chinese oak silkworm, Antheraea pernyi. - Dev. Comp. Immunol. 69: 1-11.

YANG H.X., ZHU X.R. \& LU H.S. 2002: Research progress on application of silkworm pupa in medical science. - Bull. Sci. Tech. 18: 318-322.

Youlei M., Jinghai Z., Yuntao Z., Jiaoshu L., Tianyi W., ChunFU W. \& RoNG Z. 2013: Purification and characterization of a 1,3-beta-D-glucan recognition protein from Antheraea pernyi larve that is regulated after a specific immune challenge. BMB Rep. 46: 264-269.

Yu H., Zhu B.J., Yu S., Wei G.Q., Wang L., Qian C., Abbas M.N. \& Liu C.L. 2017: Characterization and functional analysis of serpin-1 like gene from oak silkworm Antheraea pernyi. Bull. Entomol. Res. 107: 620-626.

Zhang B., Wu T.Y., Tang X.W., Zhang S.Y., Xu Q.W., Zhao Y., WANG Y.J. \& FENG C.J. 2016: Cloning, expression and characterization of Ostrinia furnacalis serpin1, a regulator of the prophenoloxidase activation system. - Comp. Biochem. Physiol. (B) 192: 9-20.

Zheng Y.P., He W.Y., Beliveau C., Nisole A., Stewart D., Zheng S.C., Doucet D., Cusson M. \& Feng Q.L. 2009: Cloning, expression and characterization of four serpin-1 cDNA variants from the spruce budworm, Choristoneura fumiferana. Comp. Biochem. Physiol. (B) 154: 165-173.

Zноu J. \& HAN D. 2006: Safety evaluation of protein of silkworm (Antheraea pernyi) pupae. - Food Chem. Toxicol. 44: 11231130.

Zou Z., Picheng Z., Weng H., Mita K. \& Jiang H. 2009: A comparative analysis of serpin genes in the silkworm genome. Genomics 93: 367-375.

Received July 10, 2017; revised and accepted September 25, 2017 Published online October 23, 2017 